Pacific

Journal of

Mathematics

VERY AMPLE LINEAR SYSTEMS ON BLOWINGS-UP AT GENERAL POINTS OF SMOOTH PROJECTIVE VARIETIES

Marc Coppens

Volume $202 \quad$ No. 2

February 2002 


\title{
VERY AMPLE LINEAR SYSTEMS ON BLOWINGS-UP AT GENERAL POINTS OF SMOOTH PROJECTIVE VARIETIES
}

\author{
Marc Coppens
}

Let $X$ be a smooth projective variety, let $L$ be a very ample invertible sheaf on $X$ and assume $N+1=\operatorname{dim}\left(H^{0}(X, L)\right)$, the dimension of the space of global sections of $L$. Let $P_{1}, \ldots, P_{t}$ be general points on $X$ and consider the blowing-up $\pi: Y \rightarrow$ $X$ of $X$ at those points. Let $E_{i}=\pi^{-1}\left(P_{i}\right)$ be the exceptional divisors of this blowing-up. Consider the invertible sheaf $M:=\pi^{*}(L) \otimes O_{Y}\left(-E_{1}-\ldots-E_{t}\right)$ on $Y$. In case $t \leq N+1$, the space of global section $H^{0}(Y, M)$ has dimension $N+1-t$. In case this dimension $N+1-t$ is at least equal to $2 \operatorname{dim}(X)+2$, hence $t \leq N-2 \operatorname{dim}(X)-1$, it is natural to ask for conditions implying $M$ is very ample on $Y$ (this bound comes from the fact that "most" smooth varieties of dimension $n$ cannot be embedded in a projective space of dimension at most $2 n$ ). For the projective plane $\mathrm{P}^{2}$ this problem

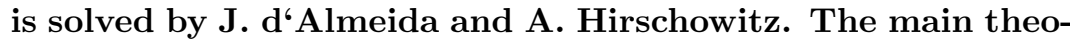
rem of this paper is a generalization of their result to the case of arbitrary smooth projective varieties under the following condition. Assume $L=L^{\prime \otimes k}$ for some $k \geq 3 \operatorname{dim}(X)+1$ with $L^{\prime}$ a very ample invertible sheaf on $X$ : If $t \leq N-2 \operatorname{dim}(X)-1$ then $M$ is very ample on $Y$. Using the same method of proof we obtain very sharp result for $K 3$-surface and let $L$ be a very ample invertible sheaf on $X$ satisfying Cliff $(L) \geq 3$ ("most" invertible sheaves on $X$ satisfy that property on the Clifford index), then $M$ is very ample if $t \leq N-5$. Examples show that the condition on the Clifford index cannot be omitted.

\section{Introduction.}

0.1. . Let $X \subset \mathbf{P}^{N}$ be a smooth projective variety of dimension $n$ defined over an algebraically closed field of characteristic 0 and assume $N \geq 2 n+2$. Let $\Lambda$ be a general linear subspace of dimension $N-(2 n+2)$ in $\mathbf{P}^{N}$. The projection with center $\Lambda$ induces an embedding $X \subset \mathbf{P}^{2 n+1}$. In general this is the best one can hope for (i.e., projecting $X$ to $\mathbf{P}^{2 n}$ in general one expects that the image of $X$ is not isomorphic to $X$ ). This embedding of $X$ in $\mathbf{P}^{2 n+1}$ is described by a linear system associated to the same invertible 
sheaf as the original embedding $X \subset \mathbf{P}^{N}$. In particular the linear system is not complete, i.e., the embedding $X \subset \mathbf{P}^{2 n+1}$ is not linearly normal. An embedding is called linearly normal if its hyperplane sections give rise to a complete linear system.

0.2. . Starting with a linearly normal embedding $X \subset \mathbf{P}^{N}$ one can try to find some linearly normal embedding in $P^{2 n+1}$ using projections from general points $P_{1} ; \ldots ; P_{N-(2 n+1)}$ on $X$. However, in order to define this morphism to $\mathbf{P}^{2 n+1}$ one has to blow-up $X$ at $P_{1} ; \ldots ; P_{N-(2 n+1)}$. Let $Y$ be the blowing-up. One can hope to obtain an embedding $Y \subset \mathbf{P}^{2 n+1}$ in this way. This would be linearly normal.

It is easy to find examples showing that this morphism $Y \rightarrow \mathbf{P}^{2 n+1}$ is not always an embedding. As an example, let $X$ be a ruled subvariety of $\mathbf{P}^{N}$. At the end of the paper, using embeddings of some special types of $K 3$-surfaces, we obtain some less trivial examples.

The obstruction for the morphism $Y \rightarrow \mathbf{P}^{2 n+1}$ to be an embedding occurs in the following situation. A general set of $N-(2 n+1)$ points of $X$ is contained in a 0 -dimensional subscheme $Z$ of $X$ of length $N-2 n+1$ such that $Z$ is a subscheme of a linear subspace $V$ of $\mathbf{P}^{N}$ of dimension $N-2 n-1$ (hence $Z$ imposes at most $N-2 n$ conditions on hyperplanes in $\mathbf{P}^{N}$ ). In that case we say that $V$ is a $(N-2 n+1)$-secant $(N-2 n-1)$-space for $X \subset \mathbf{P}^{N}$. So, the obstruction can be the existence of many such secant spaces. The main results of this paper (in particular their proofs) indicate that limited knowledge of postulation of points on curve sections of $X$ (i.e., 1 dimensional intersections of $X$ with hyperplanes of codimension $n-1$ in $\mathbf{P}^{N}$ ) gives strong information on this question. (The meaning of "limited" is the following. In principle we need knowledge on 0-dimensional subschemes of length $N-2 n+1$. It turns out that knowledge on 0 -dimensional subschemes of length $3 n+2$ is sufficient.)

0.3. . The problem considered in this paper can also be described as follows. Let $L$ be the invertible sheaf associated to some linearly normal embedding $X \subset \mathbf{P}^{N}$. Let $P_{1} ; \ldots ; P_{N-(2 n+1)}$ be general points on $X$. Let $\pi: Y \rightarrow X$ be the blowing-up of $X$ at those points $P_{i}$ and let $E_{i}=\pi^{-1}\left(P_{i}\right)$. We study very ampleness of $M:=\pi^{*}(L) \otimes O_{Y}\left(-E_{1}-\ldots-E_{N-(2 n+1)}\right)$ on $Y$.

0.4. . The main result of this paper is the following.

Theorem 1. Let $X$ be a smooth projective variety of dimension $n$; let $L^{\prime}$ be a very ample invertible sheaf on $X$ and let $L=L^{\prime \otimes t}$ for some $t \geq 3 n+1$. Let $N+1=\operatorname{dim}(\Gamma(X ; L))$ and let $P_{1} ; \ldots ; P_{N-(2 n+1)}$ be general points on $X$. Let $\pi: Y \rightarrow X$ be the blowing-up at $P_{1} ; \ldots ; P_{N-(2 n+1)}$ and for $1 \leq i \leq$ $N-(2 n+1)$ let $E_{i}=\pi^{-1}\left(P_{i}\right)$. Then $M:=\pi^{*}(L) \otimes O_{Y}\left(-E_{1}-\ldots-E_{N-(2 n+1)}\right)$ is very ample on $Y$; i.e., it defines a linearly normal embedding $Y \subset \mathbf{P}^{2 n+1}$. 
This theorem solves Conjecture 2 of my paper [7]. In case $X=\mathbf{P}^{n}$ and $L^{\prime}=O_{P^{n}}(1)$ it also gives information on Conjecture 1 of that paper. For sure, using more involved arguments, the lower bound on $t$ can be made better. Finding the best bound (or a better bound) on $t$ is interesting; in this paper I prefered to restrict to the development of a general method to prove very ampliness for the type of situation considered in this paper.

0.5. . For special types of varieties the method of the proof of Theorem 1 can be used to give much better results. As an example we discuss the case of $K 3$-surfaces. Let $X$ be a smooth $K 3$-surface and let $L$ be a very ample invertible sheaf of $X$. As explained in the first part of part 2 of this paper we have a notion of the Clifford index $\operatorname{Cliff}(L)$ of $L$. We prove the following theorem.

Theorem 2. Let $X$ be a smooth K3-surface and let $L$ be a very ample invertible sheaf of Clifford index $\operatorname{Cliff}(L) \geq 3$. Let $g+1=\operatorname{dim}(\Gamma(X ; L))$ and let $P_{1} ; \ldots ; P_{g-5}$ be general points on $X$; let $\pi: Y \rightarrow X$ be the blowingup of $X$ at $P_{1} ; \ldots ; P_{g-5} ;$ let $E_{i}=\pi^{-1}\left(P_{i}\right)$ and let $M:=\pi^{*}(L) \otimes O_{Y}\left(-E_{1}-\right.$ $\left.\ldots-E_{g-5}\right)$. Then $M$ is a very ample invertible sheaf on $Y$, in particular we obtain a linearly normal embedding $Y \subset \mathbf{P}^{5}$.

Examples show that the condition on Cliff $(L)$ in the statement cannot be omitted. Lazarsfeld proved that, in case $\operatorname{Pic}(X) \cong \mathbf{Z}$ and $L$ is the ample generator of $\operatorname{Pic}(X)$, then the curve $C$ corresponding to a general section of $L$ satisfies the results from Brill-Noether theory for linear systems on $C$ (see [15]). Hence in this case, if $g \geq 7$, one has Cliff $(L) \geq 3$ and Theorem 2 can be applied. For each value of $g$ there exist such $K 3$-surfaces and they are "general", this follows from the description of the moduli space of $K 3$ surfaces using the Torelli map (see e.g., [3]).

0.6. . Very ample linear systems on blowings-up of the projective plane are intensively studied, see e.g., [13]. In particular, the problem considered in this paper started with the paper of d'Almeida and Hirschowitz (see [9]) solving the problem for $\mathbf{P}^{2}$. Recently, De Volder and Chauvin considered similar problems on blowings-up of $\mathbf{P}^{2}$ admitting multiplicities for the exceptional divisors (see [4]). Also recently, T. Szemberg and H. Tutaj-Gasinski studied very ampleness of blowings-up of surfaces using Seshadri constants (see $[20])$.

\section{Notations and conventions.}

In this paper all varieties are defined over a fixed algebraically closed field of characteristic 0; see (1.4) for a discussion with respect to the proof of Theorem 1. A 0 -dimensional subscheme $\mathrm{Z}$ of a smooth variety $X$ is called curvilinear if there exists a smooth curve $C \subset X$ with $Z \subset C$. This is 
equivalent to $\operatorname{dim}\left(T_{P}(Z)\right) \leq 1$ for all $P \in Z$. Let $X \subset \mathbf{P}^{N}$ be a smooth $n$-dimensional projective variety. The embedding is called linearly normal if its hyperplane sections give rise to a complete linear system. A curve section (resp. surface section) of $X$ is the scheme-theoretic intersection of $X$ with a linear subspace $\Lambda$ of codimension $n-1$ (resp. $n-2$ ) such that $X \cap \Lambda$ has dimension 1 (resp. 2).

$V_{e}^{e-f}(h)$ : Set of $e$-secant $(e-f-1)$-space divisors of a linear system $h$ on a smooth curve (see (1.1.2)).

P: Set of general points on $X \subset \mathbf{P}^{N}$.

$P$ : Linear span of $\mathbf{P}$.

$T_{P}(X) ; T_{P}(Z) ; \ldots$ : Zariski tangent space.

$\langle Z\rangle$ : Linear span of some subscheme $Z \subset \mathbf{P}^{N}$; this is the intersection of all hyperplanes in $\mathbf{P}^{N}$ containing $Z$ (and it is $\mathbf{P}^{N}$ if $Z$ is not contained in some hyperplane of $\mathbf{P}^{N}$ ).

Hilb $^{k}(X)$ : Hilbert scheme of 0-dimensional subschemes of length $k$ on $X$.

$G(k, N)$ : Grassmannian of $k$-planes in $\mathbf{P}^{N}$.

$\left(\mathbf{P}^{N}\right)^{*}$ : The dual projective space (hence it is $G(N-1, N)$ ).

$D_{s}$ : Divisor on $Y$ associated to $s \in \Gamma\left(X ; L \otimes I_{\mathbf{P}}\right)$ (see (1.4)).

Cliff $(L)$ (resp. $g(L))$ : Clifford index (resp. genus) of a very ample invertible sheaf $L$ on a $K 3$-surface $X$ (see $(2.1)$ ).

$W_{d}^{r}(C)$ : Subspace of the Jacobian of a smooth curve $C$ parametrizing invertible sheaves $L$ on $C$ of degree $d$ satisfying $h^{0}(L) \geq r+1$.

\section{Part 1: Proof of Theorem 1.}

1.1.1. . Let $X$ be a smooth projective variety defined over an algebraically closed field of characteristic 0 and embedded in some projective space $\mathbf{P}^{N}$. Let $P_{1}, \ldots, P_{k}$ be $k$ general points on $X$. In case there exist two more different points $Q_{1}, Q_{2}$ on $X$ such that the $k+2$ points $P_{1}, \ldots, P_{k}, Q_{1}, Q_{2}$ belong to some $(k+2)$-secant $k$-space for $X \subset \mathbf{P}^{N}$ then the projection to $\mathbf{P}^{N-k}$ with center $P_{1}, \ldots, P_{k}$ does not give rise to an embedding into $\mathbf{P}^{N-k}$ of the blowing-up of $X$ at those points. In that case there exists a family of $(k+2)$-secant $k$-spaces for $X \subset \mathbf{P}^{N}$ of large dimension. Classifying varieties according to the existence of such family of secant spaces is a very hard problem in general. Some papers related to similar types of questions are [18] and [19]. The benefit of using the $t$-th Veronese embeddings of some original embedding of $X$ is as follows: For the new embedding of $X$ every 0 -dimensional subscheme of $X$ of length $t+1$ imposes independent conditions on hyperplanes (this can be easily seen using hyperplanes in the 
original embedding). However the dimension of the ambient projective space grows by taking such a Veronese embedding, hence for our purpose this does not look very suitable. The main ingredient in the proof of Theorem 1 solves this problem This ingredient is Proposition 1.2 in my paper [7], which is an application of the results from my paper [8]. Before restating that proposition (see (1.1.3)) let me recall some notations and definitions.

1.1.2. . Let $C$ be a smooth complete connected curve of genus $g$. Let $h$ be a linear system on $C$, let $E$ be an effective divisor of degree $e$ on $C$. We say that $E$ is an $e$-secant $(e-f-1)$-space divisor for $C$ if $\operatorname{dim}(\{D \in h \mid D$ contains $E\}) \geq \operatorname{dim}(h)-e+f$. In order to understand this notion, assume $h$ is a very ample linear system on $C$, hence it corresponds to some embedding of $C$ in $\mathbf{P}^{r}$. Then the linear span of $E$, i.e., the intersection of the hyperplanes in $\mathbf{P}^{r}$ containing $E$ (as a scheme) has dimension at most $e-f-1$.

We use the following notation: $V_{e}^{e-f}(h)$ is the set of $e$-secant $(e-f-1)$ space divisors for $h$. It is a closed subset of the $e$-th symmetric product of $C$.

1.1.3. .

Proposition (see [7], Proposition 1.2). Let $e \leq \operatorname{dim}(h)$ and let $V$ be an irreducible component of $V_{e}^{e-f}(h)$. Assume that for a general point $E$ of $V$, if $Q \in E$, then $E-Q \notin V_{e-1}^{e-f-1}(h)$. Then:

a) $3 \operatorname{dim}(V) \leq 2 e-1$ if $2 \operatorname{dim}(V) \leq \operatorname{dim}(h)+1$,

b) $\operatorname{dim}(V) \leq 2 e-2-\operatorname{dim}(h)$ if $2 \operatorname{dim}(V) \geq \operatorname{dim}(h)+1$.

This proposition will be used in the proof as follows: In case there exists, for some large value of $k$, a lot of $(k+2)$-secant $k$-planes such that the projections do not give rise to an embedding of the blowing-up of $X$ (as explained in (1.1.1)), then this already occurs for small values of $k$. Then the benefit of the Veronese embedding as explained in (1.1.1) becomes clear.

\section{2. .}

Main lemma. Consider $X \subset \mathbf{P}^{N}$ as in the statement of Theorem 1. Let $a \geq 0$ be an integer at most $N-(2 n+1)$. For general points $P_{1}, \ldots, P_{a}$ on $X$ there exists no curvilinear subscheme $Z$ of $X$ of length $a+2$ containing $P_{1}, \ldots, P_{a}$ such that $\operatorname{dim}\left(\Gamma\left(X, L \otimes I_{Z}\right)\right) \geq N-a$. (The inequalitiy $\operatorname{dim}\left(\Gamma\left(X, L \otimes I_{Z}\right)\right) \geq N-a$ would imply that $Z$ is contained in some $(a+2)$ secant a-space.)

For the reader's convenience first I give a small survey of the proof of the main lemma. We use induction on $a$. Assume for general points $P_{1}, \ldots, P_{a}$ on $X$ there exists a curvilinear subscheme $Z$ of length $a+2$ containing those points with $\operatorname{dim}(\langle Z\rangle)=a$. We consider the intersection $\langle Z\rangle \cap X$. 
First in (1.2.1) we prove that, a general curve section containing $\langle Z\rangle \cap X$ is not a smooth curve on $X$. Then in (1.2.3) we prove that this implies that $\langle Z\rangle \cap X$ contains a curve $\Lambda$ on $X$. We explain that $\Lambda$ has to be a rational normal curve, hence $X$ contains many rational normal curves. In case $X$ is a surface then the arguments in (1.2.4.2) give a contradiction. In (1.2.4.1) we prove that there exist suitable surface sections for $X$, reducing the general case to the surface case.

Proof. We are going to use induction on $a$. Since $L$ is very ample on $X$ the case $a=0$ is trivial. During the proof, we also point out that the case $a=1$ is proved without using the induction hypothesis. We write $\mathbf{P}$ to denote the set of points $\left\{P_{1}, \ldots, P_{a}\right\}$ (we also consider it as a reduced scheme). Assume that there exists a curvilinear subscheme $Z$ of $X$ of length $a+2$ containing $\mathbf{P}$ such that $\operatorname{dim}\left(\Gamma\left(X, L \cap I_{Z}\right)\right) \geq N-a$. This means, $\operatorname{dim}(\langle Z\rangle) \leq a$. Let $P$ be the linear span of $\mathbf{P}$. Since $a \leq N-(2 n+1)$ and the points $P_{1}, \ldots, P_{a}$ are general on $X$, the dimension of $P$ is $a-1$ and $P \cap X=\mathbf{P}$ as a scheme. This can be seen using the general position lemma (see e.g., [1], p. 109) using a general curve section of $X$ containing $\mathbf{P}$ (we need $a \leq \operatorname{deg}(X)$ but this inequality holds because $\operatorname{deg}(X) \geq N-n+1$ (see e.g., [11], Proposition 0 ) and we assumed $a \leq N-2 n-1)$. In particular $\operatorname{dim}(\langle Z\rangle)$ cannot be less than $a$, hence $\operatorname{dim}(\langle Z\rangle)=a$.

Let $T^{\prime} \subset \operatorname{Hilb}^{a+2}(X) \times X^{a}$ be the closure of the set of points $\left(Z ; P_{1}, \ldots, P_{a}\right)$ with $Z$ a curvilinear subscheme of $X$ of length $a+2$ satisfying $\operatorname{dim}(\Gamma(X, L \otimes$ $\left.\left.I_{Z}\right)\right) \geq N-a$ and containing the points $P_{1}, \ldots, P_{a}$ with $\mathbf{P}_{i} \neq \mathbf{P}_{j}$ for $i \neq j$. Let $T$ be an irreducible component of $T^{\prime}$ dominating $X^{a}$ (such a component exists, that's the assumption). Then $\operatorname{dim}(T) \geq$ an. Consider $I \subset T \times$ $G(N-n+1, N)$ with $\left(Z ; P_{1}, \ldots, P_{a} ; \Lambda\right) \in I$ if and only if $\langle Z\rangle \subset \Lambda$. The fibers of the projection $I \rightarrow T$ have dimension $(n-1)(N-n+1-a)$, hence $\operatorname{dim}(I) \geq$ an $+(n-1)(N-n+1-a)$. Consider the projection $\tau: I \rightarrow G(N-n+1, N)$.

\subsection{1. .}

Subclaim. For $\Lambda \in \tau(I)$ general the intersection $\Lambda \cap X$ (as a scheme) is not a smooth curve. (Notice that $\operatorname{dim}(\Lambda \cap X) \geq 1$.)

Assume for some $\Lambda \in \tau(I)$ the intersection $\Lambda \cap X$ is a smooth curve (call it $C$ ). The embedding $C \subset \Lambda$ corresponds to a linear system $g$ on $C$ of dimension $N-n+1$. Elements of $\tau^{-1}(\Lambda)$ correspond to effective divisors $Z$ on $C$ of degree $a+2$ such that $\{D \in g: D-Z \geq 0\}$ has dimension at least $N-n-a$, i.e., $Z \in V_{a+2}^{a+1}(g)$.

Since $\operatorname{dim}[G(N-n+1, N)]=(N-n+2)(n-1)$ one has $\operatorname{dim}\left(\tau^{-1}(\Lambda)\right) \geq$ $a n+(n-1)(N-n+1-a)-(n-1)(N-n+2)=(a+2)-n-1$. Let $e \leq a+2 \leq N-2 n+1$ be the integer defined as follows. For a general element of $\tau^{-1}(\Lambda)$ the divisor $Z$ contains a subdivisor $E$ of degree $e$ not imposing 
independent conditions on the linear system $g$ (hence $E \in V_{e}^{e-1}(g)$ ) while each subdivisor $E^{\prime}$ of degree $e-1$ imposes independent conditons on $g$. This implies $Z=E+P_{1}+\cdots+P_{a+2-e}$ for some points $P_{1}, \ldots, P_{a+2-e}$ on $C$. Since $Z$ is general in a subvariety of dimension at least $(a+2)-n-1$ of $V_{a+2}^{a+1}(g)$, the subdivisor $E$ is general in a subvariety of $V_{e}^{e-1}(g)$ of dimension at least $e-n-1$. So, for some $e \leq a+2 \leq N-2 n+1$ there exists some component $V$ of $V_{e}^{e-1}(g)$ with $\operatorname{dim}(V)=e-n-1+t$ for some integer $t \geq 0$ and for $E \in V$ general and $P \in E$ one has $E-P \notin V_{e-1}^{e-2}(g)$.

Now we use Proposition (1.1.3). The condition $e \leq \operatorname{dim}(g)=N-n+1$ holds. In case $2 \operatorname{dim}(V) \geq N-n+2$ we find $e-n-1+t \leq 2 e-2-(N-n+1)$ hence $e \geq N-2 n+2$, a contradiction. (Notice: Here we use the condition $a \leq N-(2 n+1)$; we should use that condition because in general the statement of Theorem 1 is sharp with respect to the upper bound on $a$.) So we are in case $2 \operatorname{dim}(V) \leq N-n+2$ and we find $3(e-n-1+t) \leq 2 e-1$, i.e., $e \leq 3 n+2-3 t \leq 3 n+2$, hence $\operatorname{dim}\left(V_{3 n+2}^{3 n+1}(g)\right) \geq 3 n+2-n-1=2 n+1$. But, because $L=L^{\prime \otimes t}$ we find that $g$ contains all sums of $t$ divisors from another very ample linear system on $C$. Since $t \geq 3 n+1$, all effective divisors on $C$ of degree $3 n+2$ impose independent conditions on $g$. This gives a contradiction, proving the subclaim.

\subsection{2. .}

Remark. We only used the assumpion $L=L^{\prime \otimes t}$ at the end of the proof of Subclaim (1.2.1). In general we proved the following lemma.

Lemma. Let $X \subset \mathbf{P}^{N}$ be a smooth $n$-dimensional variety. Let $a \leq N-$ $2 n-1$ be an integer. Assume for $\mathbf{P}=\left\{P_{1} ; \ldots ; P_{a}\right\}$ a set of general points on $X$ there exists a curvilinear subscheme $Z \subset X$ of length $a+2$ containing $\mathbf{P}$ such that $\operatorname{dim}(\langle Z\rangle)=a$. Let $\Lambda \subset \mathbf{P}^{N}$ be a general linear subspace of dimension $N-n+1$ containing $\langle Z\rangle$ and assume $\Lambda \cap X$ is a smooth curve $C$ on $X$. Let $g$ be the linear system on $C$ corresponding to the embedding $C \subset \Lambda$. Then $V_{3 n+2}^{3 n+1}(g)$ has an irreducible component of dimension at least $2 n+1$.

\subsection{3. .}

Subclaim. For $\left(Z ; P_{1}, \ldots, P_{a}\right) \in T$ general the intersection $\langle Z\rangle \cap X$ is not finite.

Take $\left(Z ; P_{1}, \ldots, P_{a}\right) \in T$ general and assume $\langle Z\rangle \cap X$ is finite. We know that $Z$ contains $\mathbf{P}$, a set of a general points on $X$. Since $P \cap X=\mathbf{P}$ as a scheme and $\operatorname{dim}(\langle Z\rangle)=a$, we know that $P$ is a hyperplane in $\langle Z\rangle$.

1.2.3.1. Assume $\langle Z\rangle \cap X$ would be curvilinear. Consider a general $\Lambda \in$ $G(N-n+1, N)$ containing $\langle Z\rangle$. Because of Bertini's Theorem, singular points of $\Lambda \cap X$ belong to $\langle Z\rangle \cap X$. For all $z \in\langle Z\rangle \cap X$ and $\Lambda \supset\langle Z\rangle$ general 
one has $\operatorname{dim}\left(\Lambda \cap T_{z}(X)\right) \leq 1$. Indeed: $\operatorname{dim}\left(T_{z}(X)(\langle Z\rangle)\right) \leq 1$ because we assume $X \cap\langle Z\rangle$ is curvilinear and $\operatorname{dim}\left(T_{z}(X)\right)=n$, hence $\{\Lambda \in G(N-$ $n+1, N)$ with $\Lambda \supset\langle Z\rangle$ and $\left.\operatorname{dim}\left(T_{z}(X) \cap \Lambda\right) \geq 2\right\}$ has dimension at most $(N-n+1-a)(n-1)-1$, while $\{\Lambda \in G(N-n+1, N)$ with $\Lambda \supset\langle Z\rangle\}$ has dimension $(N-n+1-a)(n-1)$. Since $\langle Z\rangle \cap X$ is finite it follows that $\Lambda \cap X$ is smooth for $\Lambda \in G(N-n+1, N)$ general and $\Lambda \supset\langle Z\rangle$. This contradicts Subclaim (1.2.1).

1.2.3.2. So there exists some point $Q \in\langle Z\rangle \cap X$ such that $\operatorname{dim}(\langle Z\rangle \cap$ $\left.T_{Q}(X)\right) \geq 2$. Notice that this cannot happen if $a=1$. Since $P$ is a hyperplane in $\langle Z\rangle$ also $\operatorname{dim}\left(P \cap T_{Q}(X)\right) \geq 1$. In particular since $P \cap X$ is reduced, one finds $Q \notin \mathbf{P}$. Let $P^{\prime}=\left\langle P_{1} ; \ldots ; P_{a-1}\right\rangle$. Since $P^{\prime}$ is a hyperplane in $P$ one also finds $P^{\prime} \cap T_{Q}(X) \neq \varnothing$. Let $W=\left\{P_{1} ; \ldots ; P_{a-1} ; Q\right\}$, then $\operatorname{dim}\left(\langle W\rangle \cap T_{Q}(X)\right) \geq 1$, hence $\langle W\rangle \cap X$ contains some length 2 subscheme $Z_{Q}$ with support at $Q$; its union with $\left\{P_{1} ; \ldots ; P_{a-1}\right\}$ is a curvilinear subscheme $Z^{\prime}$ of $X$ of length $a+1$. Since $\left\langle Z^{\prime}\right\rangle=\langle W\rangle$ we find that $\operatorname{dim}\left(\left\langle Z^{\prime}\right\rangle\right)=a-1$. Now we use the induction hypothesis of the main lemma, this gives a contradiction. This finishes the proof of Subclaim (1.2.3).

1.2.4. . So we find $\operatorname{dim}(\langle Z\rangle \cap X) \geq 1$. Since $P$ is a hyperplane in $\langle Z\rangle$ and $\operatorname{dim}(P \cap X)=0$ we find that, for $\left(Z ; P_{1}, \ldots, P_{a}\right) \in T$ general, $\operatorname{dim}(\langle Z\rangle \cap$ $X)=1$. Let $\Gamma$ be a 1-dimensional irreducible component of $\langle Z\rangle \cap X$. Then $\Gamma \cap P$ is a hyperplane section of $\Gamma \subset\langle\Gamma\rangle$ and it consists of $b$ points of $\left\{P_{1} ; \ldots ; P_{a}\right\}$. Those points are independent (say they are $P_{1} ; \ldots ; P_{b}$ ) hence $\operatorname{dim}(\langle\Gamma \cap P\rangle)=b-1$, so $\operatorname{dim}(\langle\Gamma\rangle)=b$ and $\Gamma$ has degree $b$. So $\Gamma$ is a rational normal curve of degree $b$. But $\Gamma$ is embedded by a linear system that contains all sums of $t$ divisors from another linear system on $\Gamma$, hence $b \geq t \geq 3 n+1$. Since $a \geq b$ we find a contradiction if $a=1$; hence the main lemma is proved in case $a=1$. So we find: For $b \geq 3 n+1$ general points $P_{1} ; \ldots ; P_{b}$ on $X$ there is a rational normal curve $\Gamma$ of degree $b$ on $X \subset \mathbf{P}^{N}$ containing those points. In case $b<a$ then for any $b$ general points $P_{1}, \ldots, P_{b}$ on $X$ we find a curvilinear subscheme $Z^{\prime}$ of length $b+2$ containing $\left\{P_{1}, \ldots, P_{b}\right\}$ with $\operatorname{dim}\left(\left\langle Z^{\prime}\right\rangle\right)=b$, a contradiction to the induction hypothesis of the main lemma, hence $b=a$. Let $C$ be the space parametrizing such curves $\Gamma$ and let $I_{1}^{\prime} \subset C \times X^{a}$ be the set of points $\left(\Gamma ; P_{1}, \ldots, P_{a}\right)$ with $P_{i} \in \Gamma$. Let $I_{1}$ be an irreducible component of $I_{1}^{\prime}$ dominating $X^{a}$. We obtain $\operatorname{dim}\left(I_{1}\right) \geq n a$. Also the projection $I_{1} \rightarrow C$ has fibers of dimension $a$. Let $U \subset C$ be the image of $I_{1}$ then $\operatorname{dim}(U) \geq a(n-1)$.

\subsubsection{1.}

Subclaim. Let $\Gamma$ be a curve corresponding to a general element of $U$. There exists $\Lambda \in G(N-n+2 ; N)$ such that $X \cap \Lambda$ is a smooth surface containing $\Gamma$.

Proof. Of course, in case $n=2$, there is nothing to prove, so assume $n>2$. Let $G(\Gamma)=\{\Lambda \in G(N-n+2, N): \Lambda \subset\langle\Gamma\rangle\}$. Since $\operatorname{dim}(\langle\Gamma\rangle)=a$, we find 
$\operatorname{dim}(G(\Gamma))=(N-n+2-a)(n-2)$. Assume there exists $Q \in\langle\Gamma\rangle \cap X$ with $\operatorname{dim}\left(T_{Q}(X) \cap\langle\Gamma\rangle\right) \geq 2$. Then one finds $a$ contradiction to the induction hypothesis of the main lemma as in (1.2.3.2). So for each $Q \in\langle\Gamma\rangle \cap X$ we find $\operatorname{dim}\left(T_{Q}(X) \cap\langle\Gamma\rangle\right) \leq 1$. Because of Bertini's Theorem, for $\Lambda \in G(\Gamma)$ general we find Sing $(\Lambda \cap X) \subset\langle\Gamma\rangle \cap X$. If $\Lambda \cap X$ would be singular at $Q \in\langle\Gamma\rangle \cap X$ then $\operatorname{dim}\left(\Lambda \cap T_{Q}(X)\right) \geq 3$. Consider $G(\Gamma ; Q)=\left\{\Lambda \in G(\Gamma): \operatorname{dim}\left(\Lambda \cap T_{Q}(X)\right) \geq 3\right\}$. Then $\operatorname{dim}[G(\Gamma ; Q)] \leq(n-2)(N-n-a+2)-2$ since $\operatorname{dim}\left(\langle\Gamma\rangle \cap T_{Q}(X)\right) \leq 1$. It follows that the union of $G(\Gamma ; Q)$ for all $Q \in\langle\Gamma\rangle \cap X$ has dimension at most $(n-2)(N-n-a+2)-1<\operatorname{dim}(G(\Gamma))$. Hence for $\Lambda \in G(\Gamma)$ general $\Lambda \cap X=S$ is a smooth surface. This finishes the proof of Subclaim (1.2.4.1).

1.2.4.2. Now, consider $I_{2} \subset G(N-n+2, N) \times U$ defined by $(\Lambda ; \Gamma) \in I_{2}$ if and only if $\Lambda \supset\langle\Gamma\rangle$. The fiber of the projection morphism $I_{2} \rightarrow U$ for a general $\Gamma \in U$ is $G(\Gamma)$, hence $\operatorname{dim}\left(I_{2}\right) \geq a(n-1)+(n-2)(N-n+2-a)$. Consider the projection morphism $\nu: I_{2} \rightarrow G(N-n+2 ; N)$. Take $\Lambda$ general in the image of $\nu$. We just proved that $\Lambda \cap X$ is a smooth surface $S$. We find $\operatorname{dim}\left(\nu^{-1}(\Lambda)\right) \geq a(n-1)+(n-2)(N-n+2-a)-(N-n+3)(n-2)=$ $a-n+2 \geq 2 n+3$. Hence $S$ is smooth surface containing a family of rational curves of dimension at least $2 n+3$, in particular each 2 points on $S$ contain a rational curve. This implies $h^{1}\left(O_{S}\right)=0$, hence those rational curves are linearly equivalent.

Take a general hyperplane section $C$ of $S \subset \Lambda$; it is a smooth curve not containing any of those rational curves. Hence those rational curves induce a linear system $h$ on $C$ of dimension at least $a-n+2$. On the other hand the degree of that linear system is a (a general hyperplane section intersects such rational normal curve at a points). Since $a-2(a-n+2)=$ $2 n-a-4 \leq 2 n-3 n-1-4=-n-5<0$, we find that the linear system $h$ is non-special on $C$ (Clifford's Theorem, see e.g., [1], p. 107). So $g(C) \leq n-2$. The linear system on $S$ defining the embedding $S \subset \Lambda$ contains the sum of $t$ divisors from another very ample linear system on $S$, hence $C$ is linearly equivalent to the sum of $t$ mutually intersecting smooth curves on $S$. Such a sum has arithmetic genus at least $(t-1)(t-2) / 2$, hence $g(C) \geq(t-1)(t-2) / 2 \geq 3 n(3 n-1) / 2$. This can be proved using the adjunciton formula: $\left(C+K_{S} \cdot C\right)=g(C)-2$ while $C$ is linearly equivalent to $C_{1}+\cdots+C_{t}$ with $\left(C_{i} \cdot C_{j}\right)>0$ and $\left(C_{i}+K_{S} . C_{i}\right) \geq-2$. So, we find $2 n-4 \geq 9 n^{2}-3 n$, hence $0 \geq 9 n^{2}-5 n+4$. This is a contradiction. This finally finishes the proof of the main lemma.

1.3. Proof of Theorem 1. We continue to use the notation $\mathbf{P}$ and $P$ introduced in the proof of the Main Lemma with $a=N-(2 n+1)$. A section $s \in \Gamma\left(X, L \otimes I_{\mathbf{P}}\right)$ will be identified with $s \in \Gamma(Y, M)$. We write $D_{s}$ to denote the divisor on $Y$. We write $E$ for the union of the exceptional divisors $E_{1}, \ldots, E_{N-(2 n+1)}$ and we identify a point $Q$ on $Y \backslash E$ with the corresponding point $Q$ on $X \backslash \mathbf{P}$. 
1.3.1. . First we prove base-point freeness. Let $Q \in Y$. If $Q \notin E$, choose $H \in\left(\mathbf{P}^{N}\right)^{*}$ with $H \supset P$ and $Q \notin H$. (Remember $P \cap X=\mathbf{P}$ as a scheme.) This hyperplane $H$ defines $s \in \Gamma\left(X, L \otimes I_{\mathbf{P}}\right)$ with $Q \notin D_{s}$.

Assume $Q \in E_{1}$. This corresponds to a tangent line $T_{Q}$ to $X$ at $P_{1}$ in $\mathbf{P}^{N}$. Since $T_{Q}$ is not contained in $P$ we can find $H \in\left(\mathbf{P}^{N}\right)^{*}$ with $P \subset H$ but $T_{Q} \not \subset H$. Then $H$ corresponds to $s \in \Gamma\left(X, L \otimes I_{\mathbf{P}}\right)$ with $Q \notin D_{s}$.

1.3.2. . Next we prove separation of points. Take $Q_{1} ; Q_{2}$, two different points on $Y$. First assume $Q_{1}$ and $Q_{2}$ are outside of $E$. Then $Z=\mathbf{P} \cup$ $\left\{Q_{1} ; Q_{2}\right\}$ is a curvilinear subscheme of length $N-2 n+1$ containing $\mathbf{P}$. Because of the main lemma we find $\operatorname{dim}(\langle Z\rangle)=\operatorname{dim} P+2$, hence there exists $H \in\left(\mathbf{P}^{N}\right)^{*}$ with $P \cup\left\{Q_{1}\right\} \subset H$ but $Q_{2} \notin H$. Then $H$ defines $s \in \Gamma\left(X, L \otimes I_{\mathbf{P}}\right)$ with $Q_{1} \in D_{s}$ but $Q_{2} \notin D_{s}$.

Assume $Q_{1} \in E_{1}$, but $Q_{2} \notin E$. The point $Q_{1}$ defines a tangent direction to $X$ at $P_{1}$, hence it defines a 0 -dimensional subscheme $Z_{Q_{1}}$ of $X$ of length 2 with support $P_{1}$. Let $Z=\mathbf{P} \cup Z_{Q_{1}} \cup\left\{Q_{2}\right\}$. It is a curvilinear subscheme of $X$ of length $N-2 n+1$ containing $\mathbf{P}$, hence $\operatorname{dim}(\langle Z\rangle)=\operatorname{dim}(P)+2$. This implies that there exists $H \in\left(\mathbf{P}^{N}\right)^{*}$ with $H \supset \mathbf{P} \cup Z_{Q_{1}}$ but $Q_{2} \notin H$. Then $H$ defines $s \in \Gamma\left(X, L \otimes I_{\mathbf{P}}\right)$ with $Q_{1} \in D_{s}$ but $Q_{2} \notin D_{s}$.

Next assume $Q_{1}$ and $Q_{2}$ both belong to $E_{1}$. Let $T_{1}$ and $T_{2}$ be the corresponding tangent lines to $X \subset \mathbf{P}^{N}$. Since $P \cap X=\mathbf{P}$ as a scheme, one finds $\operatorname{dim}\left(\left\langle P \cup T_{1} \cup T_{2}\right\rangle\right)=\operatorname{dim} P+2$. Hence there exists $H \in\left(\mathbf{P}^{N}\right)^{*}$ such that $H \supset P$ with $T_{1} \subset H$ and $T_{2} \not \subset H$. Then $H$ defines $s \in \Gamma\left(X, L \otimes I_{\mathbf{P}}\right)$ with $Q_{1} \in D_{s}$ but $Q_{2} \notin D_{s}$.

Finally assume $Q_{1} \in E_{1}$ and $Q_{2} \in E_{2}$. Let $Z_{1}$ and $Z_{2}$ be the curvilinear subschemes of length 2 on $X$ with support at $P_{1}$ and $P_{2}$ corresponding to those points. Consider $Z=Z_{1} \cup Z_{2} \cup \mathbf{P}$. It is a curvilinear subscheme of $X$ of length $N-2 n+1$ containing $\mathbf{P}$ hence the main lemma implies $\operatorname{dim}(\langle Z\rangle)=\operatorname{dim}(P)+2$. So there exists $H \in\left(\mathbf{P}^{N}\right)^{*}$ with $P \cup Z_{1} \subset H$ but $Z_{2} \not \subset H$. Then $H$ defines $s \in \Gamma\left(X, L \otimes I_{\mathbf{P}}\right)$ with $Q_{1} \in D_{s}$ but $Q_{2} \notin D_{s}$.

1.3.3. . Finally we prove separation of tangent directions. Let $Q \in Y$ and let $v \in T_{Q}(Y)$. First assume $Q \notin E$. Then $v$ corresponds to a subscheme $Z_{v}$ of length 2 of $X$ with support $Q$. Let $Z=Z_{v} \cup \mathbf{P}$. It is a curvilinear subscheme of $X$ of length $N-2 n+1$ containing $\mathbf{P}$. The main lemma implies that $\operatorname{dim}(\langle Z\rangle)=\operatorname{dim} P+2$. Hence there exists $H \in\left(\mathbf{P}^{N}\right)^{*}$ with $P \cup\{Q\} \subset H$ but $Z_{v} \not \subset H$. Then $H$ defines $s \in \Gamma\left(X, L \otimes I_{\mathbf{P}}\right)$ with $Q \in D_{s}$ but $v \notin T_{Q}\left(D_{s}\right)$.

Assume that $Q \in E_{1}$. First assume $v \in T_{Q}\left(E_{1}\right)$. Identifying $E_{1}$ with $\mathbf{P}^{n-1}$, the direction defined by $v$ corresponds to a line $L_{v}$ containing $Q$. Let $Q^{\prime}$ be another point on that line. We already know that there exists $s \in \Gamma\left(X, L \otimes I_{\mathbf{P}}\right)$ with $Q \in D_{s}$ but $Q^{\prime} \notin D_{s}$. In particular $L_{v} \not \subset D_{s}$, hence under the identification of $E_{1}$ and $\mathbf{P}^{n-1}$ the intersection of $D_{s}$ and $E_{1}$ is a hyperplane intersecting $L_{v}$ transversally at $Q$. It follows that $v \notin T_{Q}\left(D_{s}\right)$. 
So assume $v \notin T_{Q}\left(E_{1}\right)$. Then $Q$ corresponds to a subscheme $Z_{Q}$ of length 2 of $X$ and $v$ corresponds to a curvilinear subscheme $Z_{v}$ of length 3 of $X$ with support $P_{1}$ and containing $Z_{Q}$. Then $Z=Z_{v} \cup \mathbf{P}$ is a subscheme of length $N-2 n+1$ containing $\mathbf{P}$ hence the main lemma implies $\operatorname{dim}(\langle Z\rangle)=\operatorname{dim} P+2$. Hence we find $H \in\left(\mathbf{P}^{N}\right)^{*}$ with $Z_{Q} \cup \mathbf{P} \subset H$ but $Z_{v} \not \subset H$. Then $H$ defines $s \in \Gamma\left(X, L \otimes I_{\mathbf{P}}\right)$ with $Q \in D_{s}$ but $v \notin T_{Q}\left(D_{s}\right)$.

1.3.4. . In this proof we only used the fact that $\mathbf{P}$ is a set of a points on $X$ such that for each curvilinear subscheme $Z$ of length $a+2$ of $X$ containing $\mathbf{P}$ one has $\operatorname{dim}(\langle Z\rangle)=\operatorname{dim}(P)+2$. So we obtain:

Proposition. Let $X \subset \mathbf{P}^{N}$ be a smooth projective variety and let $\mathbf{P}$ be a set of a points on $X$. Let $P$ be the span of $\mathbf{P}$. Assume for all curvilinear subschemes $Z \subset X$ of length $a+2$ and containing $\mathbf{P}$ one has $\operatorname{dim}(\langle Z\rangle)=$ $\operatorname{dim}(P)+2$. Let $Y$ be the blowing-up of $X$ at $\mathbf{P}$. Then the projection of $X$ with center $P$ induces an embedding of $Y$.

1.4. . In the proof of Theorem 1 we use the characteristic zero assumption in two arguments. First of all there is the use of Bertini's Theorem. There exist Bertini Theorems for positive characteristic. Maybe they can be used causing a more involved proof and maybe a worse assumption on $t$. Next there is the use of Proposition (1.1.3). See [8], Remark 1.7 for a discussion of it. The main problem is: The linear system $g$ on the curve $C$ in the proof of Subclaim (1.2.1) need not be complete.

\section{Part 2: Proof of Theorem 2.}

2.1. . For the convenience of the reader we recall the definition of the Clifford index of a smooth curve $C$. Let $L$ be an invertible sheaf on $C$. The Clifford index of $L$ is Cliff $(L)=\operatorname{deg}(L)-2 h^{0}(L)+2$. Let $K_{C}$ be the canonical sheaf on $C$. The invertible sheaf $L$ is very special if $h^{0}(L)>1$ and $h^{0}\left(K_{C} \otimes L^{-1}\right)>1$. The Clifford index of $C$ is Cliff $(C)=\min (\{\operatorname{Cliff}(L): L$ is a very special invertible sheaf on $C\}$ ). From the Riemann-Roch Theorem it follows that in the definition of $\operatorname{Cliff}(C)$ we can restrict to line bundles with $\operatorname{deg}(L) \leq g-1$. In the proof of Theorem 2 we will use that Cliff $(C) \leq 2$ if and only if there exist integers $r \geq 1 ; d \leq g-1$ with $d-2 r \leq 2$ such that $W_{d}^{r}(C)$ is not empty.

Let $X$ be a $K 3$-surface and let $L$ be a very ample invertible sheaf on $X$. Let $C$ be a smooth curve on $X$ associated to a global section of $L$. It is proved in [12] that $\operatorname{Cliff}(C)$ is independent of the curve, so we call it the Clifford index of $L$, denoted by Cliff $(L)$. Also remember that the dimension of the complete linear system defined by $L$ is equal to the genus of $C$. We denote it by $g=g(L)$ and we consider $X \subset \mathbf{P}^{g}$ defined by $L$. 
2.2. Proof of Theorem 2. In case $g \leq 6$ it follows from the existence of special divisors (see e.g., [1], p. $206(1.1)$ ) that $W_{4}^{1}(C)$ is not empty for a smooth curve section of $X$, hence $\operatorname{Cliff}(L) \leq 2$. So, we can assume $g \geq 7$.

2.2.1. . Using (1.3.4), it is enough to prove the main lemma (1.2) in this situation. Let $t \leq g-5$ and assume for general points $P_{1} ; \ldots ; P_{t}$ on $X$ there exists a curvilinear subscheme $Z$ on $X$ of length $t+2$ containing those points such that $\operatorname{dim}(\langle Z\rangle) \leq t$ and so $\operatorname{dim}(\langle Z\rangle)=t$ follows from the general position lemma as explained in the first lines of the proof of (1.2). Since $L$ is very ample on $X$ this is not possible for $t=0$. So we use induction on $t$ and we assume $t>0$ and it is not possible to find such a subscheme $Z$ for a smaller number of general points on $X$.

2.2.2. . Let $T \subset X^{t} \times \operatorname{Hilb}^{t+2}(X)$ be the closure of the set of points $\left(P_{1}, \ldots\right.$, $\left.P_{t} ; Z\right)$ with $P_{i} \neq P_{j}$ for $i \neq j$ and $Z$ a curvilinear subscheme of length $t+2$ containing the points $P_{1}, \ldots, P_{t}$ and imposing at most $t+1$ independent conditions on $\Gamma(X ; L)$. The assumption implies that $T$ dominates $X^{t}$, let $T^{\prime}$ be a component dominating $X^{t}$. Let $I \subset T^{\prime} \times\left(\mathbf{P}^{g}\right)^{*}$ be the closure of the set of points $\left(P_{1}, \ldots, P_{t} ; Z ; H\right)$ such that $\langle Z\rangle \subset H$. Since $\operatorname{dim}(\langle Z\rangle) \leq t$, the fiber of $I$ over $\left(P_{1}, \ldots, P_{t} ; Z\right)$ has dimension at least $g-1-t$, hence $\operatorname{dim}(I) \geq t+g-1$. Assume $H \in\left(\mathbf{P}^{g}\right)^{*}$ belongs to the image of $I$, then the fiber of $H$ over $I$ has dimension at least $t-1$. Take $\left(P_{1}, \ldots, P_{t} ; Z ; H\right)$ general on $I$.

2.2.3. . Assume $H \cap X$ is a smooth curve $C$. The curve section $C \subset H$ is embedded by means of the canonical linear system on $C$. In that case secant space divisors correspond to special divisors. So, in this part of the proof, we are going to make use of the notations $W_{d}^{r}(C)$ for the subsets of the Jacobian $J(C)$ parametrizing special invertible sheaves on $C$ (see [1], Chapter IV). We find $\operatorname{dim}\left(V_{t+2}^{t+1}\left(\left|K_{C}\right|\right)\right) \geq t-1$. (Here we use $\left|K_{C}\right|$ to denote the canonical linear system on $C$, i.e., the complete linear system associated to the canonical sheaf $K_{C}$.) Let $E$ be a general element of a component of $V_{t+2}^{t+1}\left(\left|K_{C}\right|\right)$. Define the integer $\varepsilon \geq 0$ such that $E \in V_{t+2}^{t+1-\varepsilon}\left(\left|K_{C}\right|\right)$ but $E \notin V_{t+2}^{t-\varepsilon}\left(\left|K_{C}\right|\right)$. Since $t+2 \leq g$ it follows from the geometric RiemannRoch Theorem (see e.g., [1], p. 12) that $\operatorname{dim}(|E|)=\varepsilon$. Choose a subdivisor $P_{1}+\cdots+P_{\varepsilon-1}$ of $E$ such that $\operatorname{dim}\left(\left|E-P_{1}-\cdots-P_{\varepsilon-1}\right|\right)=1$. For $Q_{1} ; \ldots ; Q_{\varepsilon-1}$ general on $C$ one has $\operatorname{dim}\left(\left|E-P_{1}-\cdots-P_{\varepsilon-1}+Q_{1}+\cdots+Q_{\varepsilon-1}\right|\right)=1$ and using the geometric Riemann-Roch Theorem one finds $E-P_{1}-\cdots-P_{\varepsilon-1}+Q_{1}+$ $\cdots+Q_{\varepsilon-1} \in V_{t+2}^{t+1}\left(\left|K_{C}\right|\right)$ but $E-P_{1}-\cdots-P_{\varepsilon-1}+Q_{1}+\cdots+Q_{\varepsilon-1} \notin V_{t+2}^{t}\left(\left|K_{C}\right|\right)$. Since $E$ is a specialisation of $E-P_{1}-\cdots-P_{\varepsilon-1}+Q_{1}+\cdots+Q_{\varepsilon-1}$ one finds a contradiction unless $\varepsilon=0$. So, $\operatorname{dim}(|E|)=1$ and $|E|$ is a onedimensional subspace of $V_{t+2}^{t+1}(|K C|)$. Mapping divisors to their associated invertible sheaf we find $O_{C}(E) \in W_{t+2}^{1}(C)$, hence $\operatorname{dim}\left(W_{t+2}^{1}(C)\right) \geq t-2$. In 
case $g=7$ we have $t \leq 2$ and we find that $C$ has a linear system $g_{4}^{1}$, hence Cliff $(C) \leq 2$. Now, assume $g \geq 8$. We are going to use some dimension theorems on the varieties $W_{d}^{r}$.

First of all, if $g \geq 11$, the following is proved in [14], Theorem 2.1. Let $d$ and $r$ be integers satisfying $d \leq g+r-4$ and $r \geq 1$ and assume $\operatorname{dim}\left(W_{d}^{r}(C)\right) \geq d-2 r-2 \geq 0$. Then $W_{4}^{1}(C)$ is not empty, hence Cliff $(C) \leq 2$ (in [14] there is a list of possibilities for those curves, in all cases it is easy to find elements in $\left.W_{4}^{1}(C)\right)$. In our case, we find $\operatorname{dim}\left(W_{t+2}^{1}(C)\right) \geq(t+2)-2-2$ and $t+2 \leq g-3=g-1-2$, hence we find $\operatorname{Cliff}(C) \leq 2$ if $g \geq 11$. In case $g=8$ we find $\operatorname{dim}\left(W_{5}^{1}(C)\right) \geq 1$. In case $g=9$ (resp. $g=10$ ) we can use [6], Proposition 12: From $\operatorname{dim}\left(W_{6}^{1}(C)\right) \geq 2\left(\operatorname{resp} \cdot \operatorname{dim}\left(W_{7}^{1}(C)\right) \geq 3\right)$ it follows that $\operatorname{dim}\left(W_{5}^{1}(C)\right) \geq 1$. Hence in the cases $g=8 ; 9 ; 10$ we find the existence of a component of $W_{5}^{1}(C)$ of dimension at least 1 . In case a general element of it is of the type $L=L^{\prime}(P)$ for some $P \in C$ with $h^{0}\left(L^{\prime}\right) \geq 1$, we find $L^{\prime} \in W_{4}^{1}(C)$ hence Cliff $(C) \leq 2$. So we assume for a general such $L$ such a point $P$ does not exist (in terms of linear systems: We obtain a base point free linear system $g_{5}^{1}$ ). In those cases $C$ is birationally equivalent to a plane curve of degree 6 (for $g=10$ see [16]; for $g=11$ see [5]; for $g=8$ see [2]). The associated map from $C$ to $\mathbf{P}^{2}$ with image that plane curve of degree 6 defines an invertible sheaf $L$ belonging to $W_{6}^{2}(C)$ and again we find Cliff $(C) \leq 2$. So we conclude that $H \cap X$ is not a smooth curve $C$.

2.2.4. . Assume $\langle Z\rangle \cap X$ is a 0 -dimensional subscheme of $X$. In case it is curvilinear then for a general hyperplane $H$ containing $\langle Z\rangle$ the intersection $H \cap X$ is a smooth curve. This can be proved as in (1.2.3.1) using Bertini's Theorem. So $\langle Z\rangle \cap X$ can not be curvilinear. As explained in (1.2.3.2) we obtain a contradiction to the induction hypothesis on $t$.

2.2.5. . So we conclude that $\operatorname{dim}(\langle Z\rangle \cap X) \geq 1$. As in (1.2.4) this implies the intersection contains a rational normal curve $\Gamma$. Since $\Gamma \subset\langle Z\rangle$ and $P$ is a hyperplane in $\langle Z\rangle$, it follows that $\Gamma$ intersects $P$. Hence $\Gamma$ contains at least one of the points $P_{1} ; \ldots ; P_{t}$. Since those points are general points on $X$ it follows that a general point on $X$ is contained in a smooth rational curve on $X$. But $X$ is a $K 3$-surface, hence each smooth rational curve on $X$ is a linear system on its own. Also Pic $(X)$ is discrete and finitely generated, hence $X$ cannot have a one-dimensional family of smooth rational curves. This implies a contradiction.

2.3. . The condition Cliff $(L) \geq 3$ cannot be omitted in general.

2.3.1. . In case a general curve $C$ in the linear system associated to $L$ is trigonal (hence Cliff $(L)=1$ ) then for a general point $P$ on $X$ and a general curve $C$ through $P$ we find two more points $P_{1}$ and $P_{2}$ on $C$, such that $P+P_{1}+P_{2}$ belongs to the $g_{3}^{1}$ on $C$. Hence $P ; P_{1} ; P_{2}$ are three points on a line. Projecting with center $P$ does not give an embedding of the blowing-up $Y$ of $X$ at $P$ in $\mathbf{P}^{g-1}$. 
2.3.2. . As a second example consider the so-called Donagi-example (see [10], 2.2). Let $f: X \rightarrow \mathbf{P}^{2}$ be the double covering branched along a smooth plane sextic. Let $L=f^{*}\left(O_{\mathbf{P}^{2}}(3)\right)$, then $\operatorname{dim}(\Gamma(X ; L))=11$ and for $X \subset \mathbf{P}^{10}$ using $L$ there exists $Q \in \mathbf{P}^{10} \backslash X$ such that the projection $\pi: X \rightarrow \mathbf{P}^{9}$ with center $Q$ is the composition of $f$ and the 3 -Veronese embedding of $\mathbf{P}^{2}$. Taking $P_{1} ; P_{2}$ general on $X$ one finds $P_{1}^{\prime} ; P_{2}^{\prime}$ with $P_{i}+P_{i}^{\prime}$ a fiber of $f$ and $\left\langle P_{i} ; P_{i}^{\prime}\right\rangle \subset \mathbf{P}^{10}$ a line containing $Q$, hence $\left\langle P_{1} ; P_{1}^{\prime}\right\rangle$ and $\left\langle P_{2} ; P_{2}^{\prime}\right\rangle$ intersect. So projection with center $\left\langle P_{1} ; P_{2}\right\rangle$ does not give rise to an embedding of the blowing-up of $X$ at $P_{1}$ and $P_{2}$ in $\mathbf{P}^{8}$. A general section of $X \subset \mathbf{P}^{10}$ is isomorphic to a smooth plane curve of degree 6 (see $[\mathbf{1 0}]$ ), hence Cliff $(C)=$ 2 .

2.3.3. . One more example can be found in [17], 4.2. Let $f: X \rightarrow \mathbf{P}^{1} \times \mathbf{P}^{1}$ be a double covering branched along a smooth curve of bidegree $(4 ; 4)$ on $\mathbf{P}^{1} \times \mathbf{P}^{1}$. Let $L=f^{*}(O(2 ; 2))$, then $\operatorname{dim}(\Gamma(X ; L))=10$ and one obtains an embedding $X \subset \mathbf{P}^{9}$ using $L$. There exists $Q \in \mathbf{P}^{9} \backslash X$ such that projection $\pi: X \rightarrow \mathbf{P}^{8}$ with center $Q$ is the composition of $f$ and the embedding of $\mathbf{P}^{1} \times \mathbf{P}^{1}$ using $O(2 ; 2)$. We conclude as in the previous example finding no embedding for the blowing-up of $X$ at two general points using a projection in $\mathbf{P}^{7}$. In this example a general section of $X \subset \mathbf{P}^{9}$ has gonality 4 (see [17]); hence Cliff $(L)=2$.

\section{References}

[1] E. Arbarello, M. Cornalba, Ph. Griffiths and J. Harris, Geometry of Algebraic Curves I, Grundlehren der mathematischen Wissenschaften 267, Springer-Verlag, Berlin, 1985, MR 86h:14019, Zbl 0559.14017.

[2] E. Ballico, C. Keem, G. Martens and A. Ohbuchi, On curves of genus eight, Math. Z., 227 (1998), 543-554, MR 99b:14027, Zbl 0916.14015.

[3] W. Barth, C. Peters and A. Van de Ven, Compact Complex Surfaces, Ergebnisse der Mathematik und ihrer Grenzgebiete 3(4) Springer-Verlag, Berlin, 1984, MR 86c:32026, Zbl 0718.14023.

[4] S. Chauvin and C. De Volder, Embeddings of general blowings-up of Del-Pezzo surfaces, preprint.

[5] M. Coppens, Smooth curves having infinitely many linear systems $g_{d}^{1}$, I, Bull. Math. Soc. Belg., Sér. 40 (1988), 153-176, MR 89g:14022, Zbl 0669.14007.

[6] Some remarks on the schemes $W_{d}^{r}$, Annali di Matematica Pura ed Applicata, 157 (1990), 183-197, MR 92h:14018, Zbl 0742.14025.

[7] _ Embeddings of general blowing-ups at points, J. Reine Angew. Math., 469 (1995), 179-198, MR +97d:14008, Zbl 0833.14026.

[8] _ An infinitesimal study of secant space divisors, J. Pure Applied Algebra, 113 (1996), 121-144, MR 98d:14006, Zbl 0890.14004.

[9] J. d'Almeida and A. Hirschowitz, Quelques plongements non-spéciaux de surfaces rationelles, Math. Z., 211 (1992), 479-489, MR 94a:14012, Zbl 0759.14004. 
[10] R. Donagi and D.R. Morrison, Linear systems on K3-sections, J. Diff. Geometry, 29 (1989), 49-64, MR 90a:14046, Zbl 0626.14006.

[11] D. Eisenbud and J. Harris, On varieties of minimal degree (a centennial account), Proc. Symp. Pure Math., 46 (1987), 3-13, MR 89f:14042, Zbl 0646.14036.

[12] M. Green and R. Lazarsfeld, Special divisors on curves on K3 surfaces, Inv. Math., 89 (1987), 357-370, MR 88j:14046, Zbl 0625.14022.

[13] B. Harbourne, Very ample divisors on rational surfaces, Math. Ann., 272 (1985), 139-153, MR 86k:14026, Zbl 0563.14003.

[14] C. Keem, On the variety of special linear systems on an algebraic curve, Math. Ann., 288 (1990), 309-322, MR 91i:14024, Zbl 0692.14002.

[15] R. Lazarsfeld, Brill-Noether-Petri without degenerations, J. Diff. Geometry, 23 (1986), 299-307, MR 88b:14019, Zbl 0608.14026.

[16] G. Martens, Eine charakterizierung glatter ebener kurven, Archiv der Math., 41 (1983), 37-43, MR 84m:14010, Zbl 0511.14016.

[17] _ On curves on K3 surfaces, L.N.M., 1389 (1989), 174-182, MR 90m:14026, Zbl 0698.14036.

[18] Z. Ran, Local differential geometry and generic projections of threefolds, J. Diff. Geometry, 32 (1990), 131-137, MR 91g:14034, Zbl 0788.14037.

[19] _ The (dimension + 2)-secant lemma, Inv. Math., 106 (1991), 65-71, MR 92j:14055, Zbl 0767.14024.

[20] T. Szemberg and H. Tutaj-Gasinska, Embeddings of general blowups of abelian surfaces, Manuscripta Math., 103(2) (2000), 183-190, MR 2001k:14039.

Received April 5, 2000 and revised September 7, 2000. The author is affiliated with the University of Leuven as a research fellow.

Katholieke Hogeschool Kempen

Departement Industrieel Ingenieur en BiotechnieK

Campus H.I. Kempen

KLEINHOEFSTRAat 4

B 2440 Geel Belgium

E-mail address: marc.coppens@khk.be 